\title{
Human embryonic stem cells-derived endothelial cell therapy facilitates kidney regeneration by stimulating renal resident stem cell proliferation in acute kidney injury
}

\author{
JIA XiaoHua ${ }^{1,2}$, LÜ He $^{3}$, LI Chen ${ }^{4}$, FENG GuoWei ${ }^{5}$, YAO XinPeng ${ }^{1}$, MAO LiNa $^{1}$, KE Ting Yu $^{1}$, \\ CHE YongZhe ${ }^{1,2}$, XU Yong ${ }^{5}$, LI ZongJin ${ }^{1,2^{*}} \&$ KONG DeLing ${ }^{1 *}$ \\ ${ }^{1}$ State Key Laboratory of Medicinal Chemical Biology, Key Laboratory of Bioactive Materials of Ministry of Education, Nankai University, \\ Tianjin 300071, China; \\ ${ }^{2}$ School of Medicine, Nankai University, Tianjin 300071, China; \\ ${ }^{3}$ College of Basic Medicine, Yanbian University, Yanji 133000, China; \\ ${ }^{4}$ Diabetes Research Group, King's College London, WC2R 2LS, UK; \\ ${ }^{5}$ Department of Urology, Second Hospital of Tianjin Medical University, Tianjin Institute of Urology, Tianjin 300071, China
}

Received October 9, 2012; accepted November 19, 2012; published online June 3, 2013

\begin{abstract}
Endothelial cell therapy has been implicated to enhance tissue regeneration and vascularization in ischemic kidney. However, no published study has yet examined direct effects of endothelial cell treatment in kidney recovery. This study investigated the therapeutic efficacy of endothelial cells in a mouse model with acute kidney injury (AKI). Thus, human embryonic stem cells-derived endothelial cells (hESC-ECs) labeled with a reporter system encoding a double fusion reporter gene for firefly luciferase (Fluc) and green fluorescent protein (GFP) were characterized by Fluc imaging and immunofluoresence staining. Cultured hESC-ECs $\left(1 \times 10^{6}\right)$ were injected into ischemic kidney shortly after AKI. Survival of the transplanted hESC-ECs was monitored in vivo from day 1 to 14 after endothelial cell transplantation and potential impact of hESC-EC treatment on renal regeneration was assessed by histological analyses. We report that a substantial level of bioluminescence activity was detected $24 \mathrm{~h}$ after hESC-EC injection followed by a gradual decline from 1 to $14 \mathrm{~d}$. Human ESC-ECs markedly accelerated kidney cell proliferation in response to ischaemia-induced damage, indicated by an elevated number of $\mathrm{BrdU}^{+}$cells. Co-expression of Sca-1, a kidney stem cell proliferation marker, and BrdU further suggested that the observed stimulation in renal cell regeneration was, at least in part, due to increased proliferation of renal resident stem cells especially within the medullary cords and arteriole. Differentiation of hESC-ECs to smooth muscle cells was also observed at an early stage of kidney recovery. In summary, our results suggest that endothelial cell therapy facilitates kidney recovery by promoting vascularization, trans-differentiation and endogenous renal stem cell proliferation in AKI.
\end{abstract}

molecular imaging, kidney ischemia, renal stem cells, endothelial cells, cell transplantation

Citation: Jia X H, Lü H, Li C, et al. Human embryonic stem cells-derived endothelial cell therapy facilitates kidney regeneration by stimulating renal resident stem cell proliferation in acute kidney injury. Chin Sci Bull, 2013, 58: 2820-2827, doi: 10.1007/s11434-013-5890-3

Renal failure is among the highest causes of mortality in hospitalized patients [1,2]. Acute ischemic kidney injury is considered the leading cause of acute renal failure, which may progress and lead to onset of chronic kidney disease and renal failure. Prolonged kidney malfunction is contributed primarily by post-ischemic endothelial cell dysfunction

*Corresponding authors (email: zongjinli@nankai.edu.cn; kongdeling@nankai.edu.cn) and microvascular impairment. Endothelial cell administration, which improves revascularization, especially functional microvasculature, has therefore been proposed as a promising therapeutic solution.

In the past decade, considerable research efforts have focused on the development of cell-based therapies against tissue damage, ranging from autologous transplantation of adult stem cells [3], bone marrow- and peripheral blood- 
derived endothelial progenitor cells (EPCs) [4-6], to cotransplantation of human endothelial cells with smooth muscle progenitors [7] or transplantation of mesenchymal stem cells (MSCs) [8-10]. However, autologous transplantation of adult stem cells and EPCs is limited by shortage of cell supply and metabolic complications of the patients [11]. Human embryonic stem cells (hESCs), with its pluripotency and the capacity to differentiate into selective cell types in vitro, provide an alternative cell source to advance cellbased therapy. Three hESC approaches have been approved by the FDA to date [7]. In particular, the hESC-derived endothelial cells (hESC-ECs) have been extensively examined $[12,13]$, due to its positive impact on angiogenesis and vasculogenesis observed in myocardial ischemia, cerebrovascular and peripheral vascular diseases [14-18].

In kidney ischemia, a facilitative role of hESC-ECs has also been implicated, but no report investigated the efficacy of hESC-EC transplantation in AKI. Previous studies mainly assessed the capacity of hESC-ECs integration during revascularization [19], but increasing evidence have suggested the possibility of differentiation-independent mechanisms of hESC-ECs in ischemic organ recovery [20,21]. Given that the molecular mechanisms by which hESC-ECs facilitate ischemic renal regeneration are largely unknown, the present study investigated the role of hESC-ECs in renal repairing after AKI both in vitro and in vivo. We observed that hESC-EC transplantation accelerated renal repairing through cell-autonomous effects, which stimulated transdifferentiation of hESC-ECs into smooth muscle cells, facilitated vascularization at the damaged site, and ameliorated proliferation of resident stem cells in kidney.

\section{Materials and methods}

\subsection{Human ESC-ECs}

Human ESC-ECs were obtained and cultured as previously described [14,15], and were transfected with a self-inactivating lentiviral vector, constructed with a ubiquitin promoter-driven firefly luciferase and an enhanced green fluorescence protein (Fluc-eGFP) double fusion reporter gene $[14,22]$. The transfected hESC-ECs were maintained in EGM-2 medium (Lonza, Basel, Switzerland) under standard cell culture conditions $\left(37^{\circ} \mathrm{C}, 95 \% \mathrm{O}_{2} / 5 \% \mathrm{CO}_{2}\right)$ for $3 \mathrm{~d}$ prior to experimental use.

\subsection{Acute kidney ischemia (AKI)}

All procedures were performed according to the Regulations for the Administration of Affairs Concerning Experimental Animals (Tianjin, revised in June 2004). Female SCID beige mice were purchased from the Laboratory Animal Centre of The Academy of Military Medical Sciences (Beijing, China) and maintained under standard conditions. At 8 weeks of age, mice were anaesthetized with $300 \mathrm{mg} / \mathrm{kg}$ chloraldurat injected intraperitoneally, and a flank incision on left kidney was made. For unilateral ischemia/reflow (I/R), the left renal pedicle was clamped using a vascular clamp. Mouse abdomen was covered with gauze moistened in saline throughout the procedure while the animal was maintained at $37^{\circ} \mathrm{C}$ using a warming pad. After $30 \mathrm{~min}$, the clamp was removed and reperfusion was confirmed visually. At specified time points, mice were sacrificed and kidney tissues were either snap frozen in liquid nitrogen and embedded in OCT (Miles Scientific) or fixed in $4 \%$ polyoxymethylene and embedded in paraffin.

\subsection{Experimental protocols}

After AKI procedure was carried out, mice were assigned to one of following groups ( $n=10$ in each group): the ECs group, in which mice received the injections into renal outer medulla at three injection sites with a total of $1 \times 10^{6}$ ESC-ECs in $30 \mu \mathrm{L}$ saline; the ECs+M group, in which mice received the same amount of hESC-ECs co-injected with Matrigel (BD Biosciences); the control (Saline) group, in which each mice received an injection of saline; and Sham-operated group, in which mice were subjected to the same surgical procedure of AKI without clamping the renal vessels. For cell proliferation measurement, mice were i.p. injected with $100 \mathrm{mg} / \mathrm{kg}$ BrdU (Sigma, $10 \mathrm{mg} / \mathrm{mL}$ in saline) $18 \mathrm{~h}$ before the animals were sacrificed.

\subsection{Optical bioluminescence imaging}

Bioluminescence imaging (BLI) was performed using the Xenogen IVIS Lumina II system as detailed previously [23]. After i.p. injection of reporter probe D-Luciferin (150 $\mathrm{mg}$ of luciferin $/ \mathrm{kg}$ ), the animals were imaged for $2 \mathrm{~s}$ to $2 \mathrm{~min}$ and the same procedure was repeated at the specified time points up to $14 \mathrm{~d}$. The imaging signals were quantified in units of maximum photons per second per square centimeter per steridian. Bioluminescence images were taken by an investigator unaware of the study design to remain unbiased.

\subsection{Histology and immunohistochemistry}

At days 3 and 14 after I/R, mice were euthanized and the left kidneys were thoroughly perfused with saline to remove blood from the vascular beds. Kidneys were then resected from the abdominal cavity and extraneous tissues were cleared off. Kidney specimens were embedded in paraffin or OCT compound, and cut into $5 \mu \mathrm{m}$ sections, which were subsequently examined by hematoxylin-eosin staining and immunofluoresence staining. To track $\mathrm{GFP}^{+}$hESC-ECs in kidneys, rabbit anti-GFP antibody (Invitrogen), mouse monoclonal smooth muscle actin (SMA) antibody (Boster, China), monoclonal anti-mouse Sca-1 antibody (Cedarlane), and mouse monoclonal halogenated pyrimidine thymidine 
analog bromodeoxyuridine (BrdU) antibody (Santa Cruz) were used. Alexa Fluor 488 and Alexa Fluor 594-conjugated secondary antibodies were applied appropriately. DAPI was used for nuclear counterstaining. To examine vascular density in infarction sites, rat anti-mouse CD31 (BD Pharmingen) and mouse SMA antibodies were used. The number of capillary vessels and microvessels were counted by an unbiased investigator in ten randomly selected high-power fields (HPF) using a fluorescence microscope at $400 \times$ magnitude. For cell proliferation quantification, the sections were incubated with mouse monoclonal BrdU antibody at $4{ }^{\circ} \mathrm{C}$ overnight before histological analyses.

\subsection{Examination of renal functions}

Plasma urea (BUN) and Creatinine were measured with a COBAS automatic biochemistry analyzer (Roche).

\subsection{Statistical analysis}

All data are expressed as mean \pm SEM. One-way ANOVA analysis of variance was employed for comparing differences between 2 groups. To assess the significance of differences among more than 2 groups, least significant difference (equal variances) and Dunnett's T3 (non-equal variances) post hoc tests were used. All tests were two-tailed, and significance was accepted at $P<0.05$.

\section{Results}

\subsection{Characterization of hESC-ECs}

The hESC-ECs were first characterized by assessments of luciferase activity and GFP expression. A linear association was established between bioluminescence activity of luciferase (BLI; photons $\mathrm{sec}^{-1} \mathrm{~cm}^{-2} \mathrm{sr}^{-1}$ ) and the amount of hESC-ECs $\left(r^{2}>0.99\right.$; data not shown), indicating that luciferase signal can be used as a reliable parameter for hESCECs quantification. GFPs were also stably incorporated into hESC-ECs, indicated by positive green fluorescence (data not shown). These observation are consistent with previous studies, in which minimal effects of reporter gene transfection on hESC-ECs survival, proliferation, and differentiation were reported $[14,17,24]$.

\subsection{Localization and survival of hESC-ECs in ischemic kidney}

Human ESC-ECs were injected to the parenchyma of the ischemic kidney with and without Matrigel, and monitored by BLI for 14 d (Figure 1(a), without Matrigel; Figure 1(b), with Matrigel). In both hESC-ECs-treated groups (Figure 1(a),(b)), high levels of luciferase signal were detected immediately after transplantation (day 0 ). In contrast, no signif- icant bioactivity was observed from the control group (data not shown). From day 1 to day 14, continuous decline of bioluminescence were observed from both hESC-ECs-injected groups, corresponding to rapid cell loss during this time period. Indeed, quantitative analyses revealed that only less than $2.3 \%$ survival activity of hECS-ECs could be detected in vivo at day 9 (Figure 1(c)). In addition, co-injection of hESC-ECs with Matrigel resulted in increase in cell retention in the first two days after transplantation (10.4 $\pm 1.7 \times 10^{4}$ photons $\mathrm{sec}^{-1} \mathrm{~cm}^{-2} \mathrm{sr}^{-1}$ vs $7.6 \pm 0.8 \times 10^{4}$ photons $\mathrm{sec}^{-1} \mathrm{~cm}^{-2} \mathrm{sr}^{-1}$, BLI values obtained $12 \mathrm{~h}$ after injection for hESC-EC- and Matrigel-treated animals vs without Matrigel, $n=8, P=0.016$ ).

\subsection{Neovasculature formation}

To assess the angiogenic potential of hESC-ECs, ischemic kidneys were examined for vascular density at day 14 after hESC-ECs transplantation. Immunostaining of kidney sections for endothelial cell marker CD31 (Figure 2(a), shown in red) displayed higher capillary density at hESC-EC injected sites regardless of the presence of Matrigel, although the increase is not statistically significant $(34 \pm 3.4$ capillaries/HPF vs 31.3 \pm 3.5 , hESC-ECs without Matrigel vs salinetreated group, $n=8, P=0.27$; Figure 2(a),(b)). Co-injection of hESC-ECs with Matrigel enhanced vascularture formation, shown by a significant elevation in capillary density (46 \pm 3.3 capillaries/HPF vs $34 \pm 3.4$, hESC-EC with Matrigel vs hESC-ECs without Matrigel, $n=8, P=0.037$; Figure 2(a),(b)). We also evaluated effects of hESC-EC administration on mature vessel formation by assessing the expression levels of SMA, a marker of mature blood vessels. Increased SMA expression was detected in kidneys from hECS-ECs-treated animals (Figure 2(a),(c)). Co-localization of ECs and SMA-positive staining was mostly located in mature blood vessels (Figure 2(d); GFP positive cells in green, SMA in red, white arrows), suggesting that hESC-ECs may involve repair of blood vessels.

\subsection{Human ESC-ECs promote endogenous renal cell proliferation}

Impact on cell proliferation of hESC-EC administration was investigated by BrdU incorporation. Immunochemical results illustrated significant increase of $\mathrm{BrdU}^{+}$proliferating cells in kidneys of hESC-ECs-treated groups in comparison to the control group $(94.0 \pm 2.5$ cells/HPF vs $78.5 \pm 6.4$ vs 37.5 \pm 3.3 , hESC-ECs with Matrigel vs hESC-ECs without Matrigel vs saline-treated group; Figure 3). Double immunostaining with Sca-1, a stem cell factor-receptor, and BrdU revealed the elevated level of proliferating endogenous renal stem cells in hESC-ECs-treated kidneys (Figure 4(a)); Sca-1 in red, BrdU in green). Sca- $1^{+}$cells are present in sham-operated kidneys, but none of these cells are in dividing status. A few Sca-1/BrdU double positive cells 

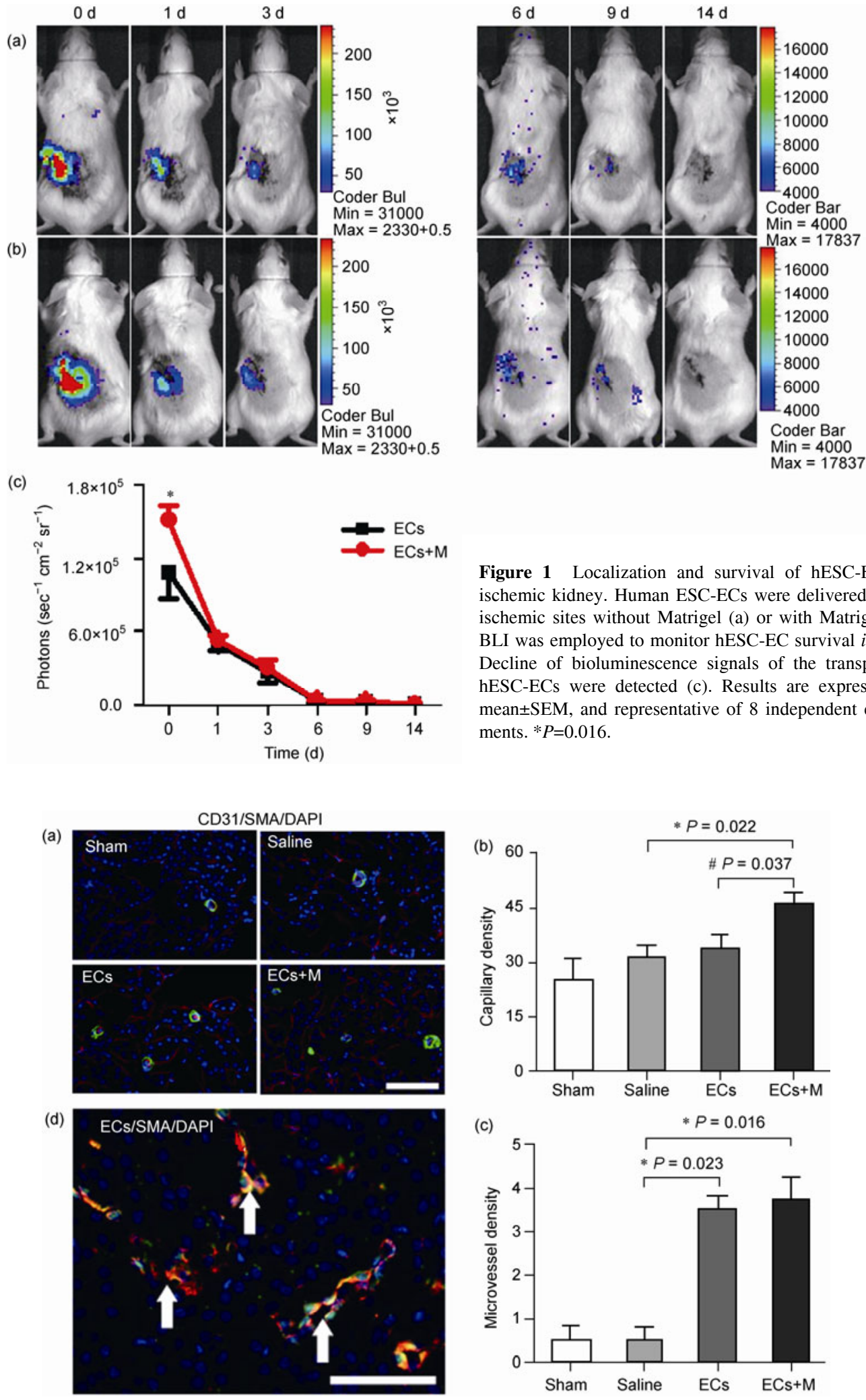

Figure 2 Human ESC-ECs increase vascular density. (a) Immunohistochemical staining of CD31 (red) and SMA (green) in ischemic kidney sections 14 days in hESC-EC-treated group (ECs), hESC-EC- and Matrigel-treated group (ECs+M), saline-treated control group (Saline) and Sham-operated group (Sham). (b) Quantitative analysis revealed a larger number of CD31 $1^{+}$capillaries in the $\mathrm{hESC}$-ECs Matrigel co-injected group in comparison to the $\mathrm{hESC}$-ECs only and saline-treated control groups. $* P=0.022$, vs saline-treated control group; $\# P=0.037$, vs hESC-EC-treated group without Matrigel. $n=8$. (c) Human ESC-ECs increased microvessel formation. $* P=0.016$, vs saline-treated control group; $* P=0.023$, vs saline-treated control group. $n=8$. (d) At day 3 after hESC-EC injection, the transplanted hESC-ECs were identified as green (GFP positive) which differentiate into smooth muscle cells (SMA in red) (arrow). Nuclei were stained with DAPI (blue). Scale bar $=50 \mu \mathrm{m}$. Results are expressed as mean \pm SEM and representative of 8 independent experiments. 
(a)

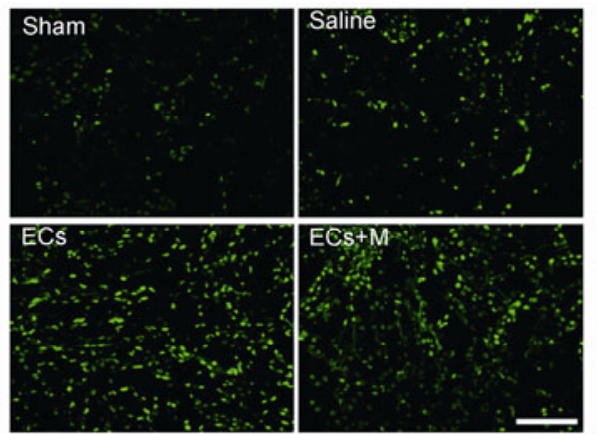

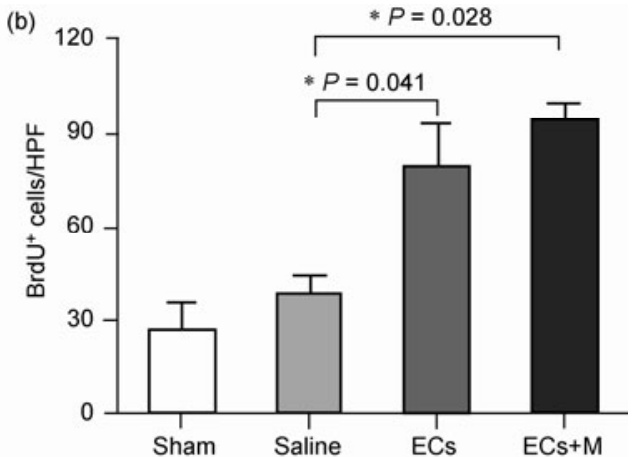

Figure 3 Human ESC-ECs increase endogenous renal cell proliferation. (a) BrdU incorporation was used to investigate cell proliferation in ischemic kidneys of the hESC-EC-treated group (ECs), hESC-EC- and Matrigel-treated group (ECs+M), saline-treated control group (Saline) and Sham-operated group (Sham). Scale bar=50 $\mu \mathrm{m}$. (b) At day 14 after AKI, increased numbers of proliferating cells were observed in kidneys of the hESC-ECs-treated groups. Results are expressed as mean \pm SEM and representative of 8 independent experiments. $* P=0.041$, ECs vs saline; $P=0.028$, ECs + Matrigel vs saline. $n=8$.

(a)
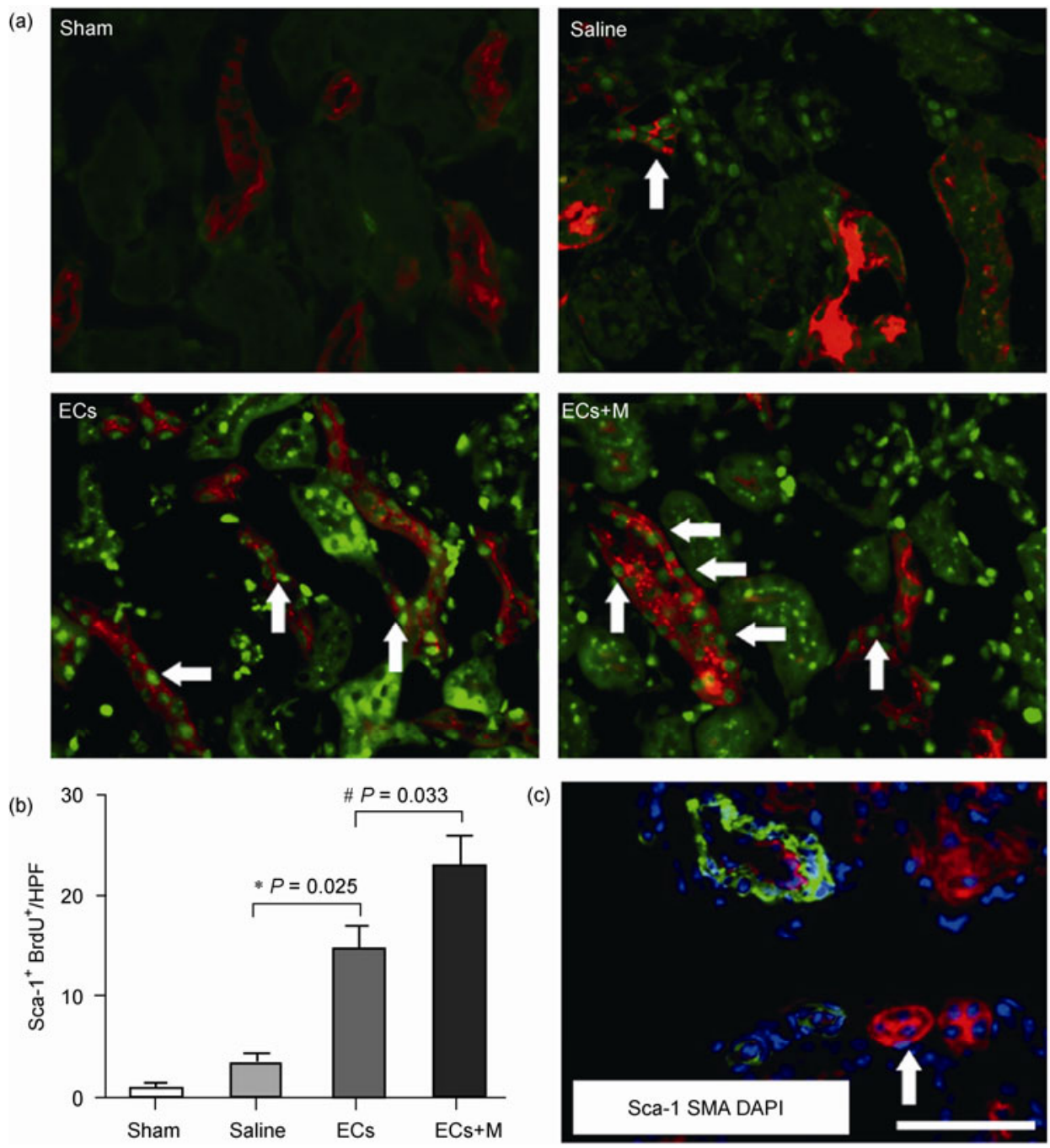

Figure 4 Human ESC-ECs increase cell proliferation via stimulating endogenous renal resident stem cells. (a) Frozen sections of mouse kidneys were immunostained with anti-mouse Sca-1(red) and BrdU (green). A large proportion of Sca- $1^{+}$cells co-express BrdU (arrow) in both ECs-treated kidneys. (b) Graph depicting the contribution of renal precursors following exogenous administration of hESC-ECs during renal repair after AKI. $14 \mathrm{~d}$ following AKI, hESC-ECs activate endogenous expansion of Sca- $1^{+}$renal stem cells. The number of Sca- $1^{+}$cells co-expressing BrdU is much greater in hESC-ECs versus non-ECs treated kidneys. (c) In situ immunofluorescence staining of Sca-1 (red) and SMA (green) illustrate localization of Sca- $1^{+}$renal stem cells in the medullary cords and arteriole (arrow). Nuclei were stained with DAPI (blue). Scale bar $=50 \mu \mathrm{m}$. Results are expressed as mean \pm SEM and representative of 8 independent experiments. $* P=0.025$ vs saline-treated control group; $\# P=0.033$ vs hESC-EC-treated group without Matrigel. $n=8$. 
were located in the saline-treated kidneys. In contrast, a large number of $\mathrm{Sca}^{-}{ }^{+}$cells co-express BrdU (arrow) in both ECs-treated kidneys (white arrows). Subsequent quantitative assessment of proliferating $\mathrm{Sca}-1^{+}$renal stem cells reported a 4.6-fold increase in hESC-ECs-treated animals $(14.7 \pm 2.2$ cells/HPF vs $3.2 \pm 1.0$, hESC-ECs without Matrigel vs saline-treated group, $n=8, P=0.025$; Figure 4(b)). Moreover, co-injection of hECS-ECs with Matrigel led to a significant elevation of proliferating Sca- $1^{+}$cells $(22.8 \pm 3.1$ cells/HPF vs $14.7 \pm 2.2$, hESC-ECs with Matrigel vs without Matrigel, $n=8, P=0.033$ ). Co-staining of Sca-1 and SMA demonstrated that the renal progenitor cells were mainly localized within the medullary cords and arteriole (Figure 4(c); Sca-1 in red; SMA in green).

\subsection{Renal function and morphology}

We have also examined kidney sections from all 4 study groups for potential morphological changes of acute tubular necrosis (Figure 5(a)). No renal damage was observed from Sham-operated kidney sections. In saline-treated group, typical I/R-induced damage was observed, characterized by tubular dilatation and necrosis, epithelial cell shedding, inflammatory cell infiltration, cast formation and cortical necrosis. In both hESC-ECs-treated groups, with and without Matrigel, I/R-resulted lesions were decreased compared to saline-treated group and the renal tubules and glomeruli remained as normal histological structure. For kidney function assessment, we quantified BUN and plasma creatinine levels and found no significant difference among the four groups (Figure 5(b),(c)), suggesting that I/R did not cause significant changes of renal function 14 days after treatment (plasma concentrations of BUN: Sham, $8.8 \pm 1.5 \mathrm{mmol} / \mathrm{L}$;
Saline-treated, $8.1 \pm 1.2 \mathrm{mmol} / \mathrm{L}$; hESCs-EC-treated, 7.6 \pm 1.4 $\mathrm{mmol} / \mathrm{L}$; hESC-ECs co-injected with Matrigel, 7.9 \pm 0.6 $\mathrm{mmol} / \mathrm{L}, n=8, P>0.05)$.

\section{Discussion}

It has been shown that endothelial cell therapy facilitated recovery of glomerular and tubular compartments in AKI $[19,25]$ and that systemic administration of human endothelial cells led to structural and functional restoration of damaged kidney [26]. Since molecular mechanisms by which hESC-ECs stimulate renal regeneration are not yet fully understood, we investigated effects of direct administration of hESC-ECs in mice with AKI. One of the major challenges of cell-based therapies is that the currently available cell tracing methods still lack sufficient sensitivity and specificity to trace and monitor cell survival and engraftment. In this study, we employed a non-invasive bioluminescence imaging technique $[14,15]$ to track and measure the survival of hESC-ECs in vivo. In addition, it has been shown that cells co-injected with Matrigel exhibited enhanced adherence, migration and formation of capillary-like structure [27], so we also assessed the effectiveness of Matrigel in cell delivery. Our results indicate that direct hESCECs injection at ischemic sites facilitated renal recovery via hESC-EC-induced improvements on neovascularization, endothelial cell trans-differentiation and endogenous renal cell proliferation. Co-injection with Matrigel enhanced hECS-EC survival and revascularization, in agreement with earlier reports where therapeutic potentials of Matrigel were implicated [27].

To elucidate the molecular mechanisms involved in

(a)



(b)



Saline

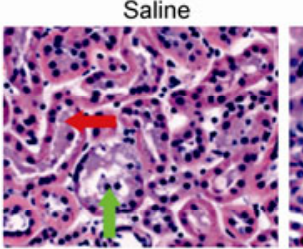

ECs

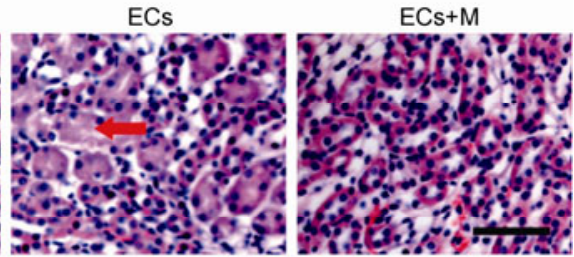

(c)

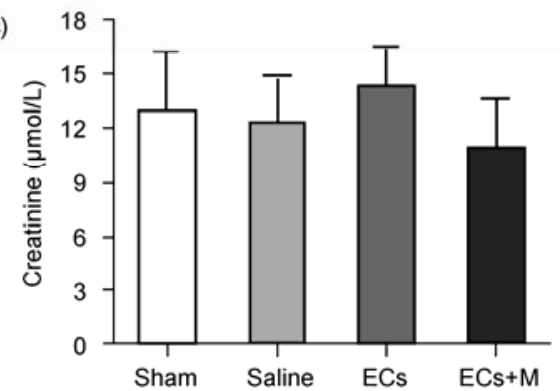

Figure 5 Morphological analysis, blood urea nitrogen (BUN) and plasma creatinine levels. (a) No renal damage was observed in sham-operated group; Typical renal damage including tubular dilatation and necrosis, epithelial cell shedding, inflammatory cell infiltration, cast formation and cortical necrosis was present in saline-treated group (arrows); In the both ECs-treated groups, the extent of lesions was decreased as compared to saline group. Scale bar=50 $\mu \mathrm{m}$. At day 14, BUN (b) and plasma creatinine (c) values were not significantly different among the four groups. 
hESC-EC-induced kidney regeneration, we first investigated the effects of hESC-ECs on angiogenesis and mature vessel formation. Despite the diminishing hESC-EC bioactivity, expression of SMA, a mature blood vessel marker, increased significantly in hESC-ECs-treated kidneys $14 \mathrm{~d}$ after hESC-ECs injection, indicative of enhanced capillary formation. Co-localization of SMA and CD31, a human endothelial cell marker, provided evidence of hESC-EC differentiation into smooth muscle cells and their direct involvement in angiogenesis and their differentiation into smooth muscle cells. Indeed, endothelial cell trans-differentiation into smooth muscle cells, a process known as endothelial-mesenchymal transition (EnMT), has been demonstrated both in vitro and in vivo $[28,29]$. However, previous studies also reported differentiation-independent mechanisms of EPCs in facilitating renal regeneration [21].

To investigate whether the beneficial effects of hECS-EC in kidney recovery may be through direct impact of hECSECs on renal cell renewal, BrdU staining was adopted to study the effect of hESC-ECs on renal cell proliferation. We observed a significant increase in the number of $\mathrm{BrdU}^{+}$cells, indicating a positive role of hESC-ECs on kidney cell regeneration. It is well established that the renal resident stem cells are essential in kidney development and regeneration [30-32]. The non-tubular Sca- $1^{+}$cells, in particular, have shown protective properties against kidney injury, which may be partly due to their ability to supply angiogenic, anti-apoptotic, and mitogenic factors during renal regeneration [33]. We found that $14 \mathrm{~d}$ after hESC-ECs injection, expression level of Sca- $1^{+}$was significantly increased. Furthermore, co-expression of BrdU and Sca-1 by cells within the medullary cords and arteriole suggested that hESC-ECs interact with endogenous Sca- $1^{+}$renal progenitor cells and promote resident stem cell proliferation.

We observed no detrimental implications of hESC-ECs transplantation. Standard histological structures of renal tubules and glomeruli were obtained from hESC-ECstreated kidneys, in addition to normalized clearance rate of blood urea (BUN) and creatine, indicating an absence of significant renal function impairment following hESC-EC treatment. These results, along with several other reports $[19,21]$, reinstate the therapeutic potential of endothelial cell administration against AKI.

One question remaining is that it is still unclear whether cell-based therapies restore structure and/or function of damaged organ via direct effects per se, since paracrine effects have also been proposed by studies using adult stem cell therapy and MSC transplantation [7]. Briefly, mediators such as cytokines and growth factors secreted by the progenitor cells induce angiogenesis and resident stem cell activation in recipient tissues [20,34]. Results presented in this study illustrated that the ameliorating effects of hECS-EC treatment in kidney regeneration could be partly explained by hECS-EC-induced revascularization and resident stem cell proliferation. We have also shown that, although the bioluminescence of hESC-ECs was gradually lost following hESC-ECs transplantation, renal resident stem cell proliferation increased significantly in hESC-ECs-treated kidneys in the absence of bioactive hESC-ECs. The enhanced proliferative activity of endogenous renal stem cells may be due to hESC-EC-stimulated innate repair cascades, which remained activated even when the bioactivity of hESC-ECs became undetectable. Future studies are required to identify potential factors that are regulated by hESC-ECs and stimulate endogenous renal repairing in a nonautonomous manner.

In summary, we have demonstrated that bioluminescence imaging technique is a reliable approach to examine cell survival and interactions between transplanted hESC-ECs and endogenous renal cells in vivo. It is also worth noting that hESC-EC treatment significantly stimulated kidney repairing via direct effects on revascularization, vessel formation and renal resident stem cell proliferation.

This work was supported by the National Key Scientific Program of China (2011CB964903), the National Natural Science Foundation of China (31071308, 30970746, 81220108015), and Doctoral Fund of Ministry of Education of China (20090031110024). FP7-PEOPLE-IRSES project ABREM is also acknowledged.

1 Patschan D, Patschan S, Muller G A. Endothelial progenitor cells in acute ischemic kidney injury: Strategies for increasing the cells' renoprotective competence. Int J Nephrol, 2011, 828369

2 Munshi R, Hsu C, Himmelfarb J. Advances in understanding ischemic acute kidney injury. BMC Med, 2011, 9: 11

3 Strauer B E, Kornowski R. Stem cell therapy in perspective. Circulation, 2003, 107: 929-934

4 Brodsky S V, Gao S, Li H, et al. Hyperglycemic switch from mitochondrial nitric oxide to superoxide production in endothelial cells. Am J Physiol Heart Circ Physiol, 2002, 283: H2130-2139

5 Yamamoto T, Tada T, Brodsky S V, et al. Intravital videomicroscopy of peritubular capillaries in renal ischemia. Am J Physiol Renal Physiol, 2002, 282: F1150-1155

6 Kumar A H, Caplice N M. Clinical potential of adult vascular progenitor cells. Arterioscler Thromb Vasc Biol, 2010, 30: 1080-1087

7 Cheung C, Sinha S. Human embryonic stem cell-derived vascular smooth muscle cells in therapeutic neovascularisation. J Mol Cell Cardiol, 2011, 51: 651-664

8 Morigi M, Rota C, Montemurro T, et al. Life-sparing effect of human cord blood-mesenchymal stem cells in experimental acute kidney injury. Stem Cells, 2010, 28: 513-522

9 Liu Y, Ye Z, Wang Y, et al. Induction-dependent neural marker expression and electrophysiological characteristics of bone marrow mesenchymal stem cells that naturally express high levels of nestin. Chin Sci Bull, 2011, 56: 640-646

10 Li D, Wang W, Guo R, et al. Restoration of rat calvarial defects by poly(lactide-do-glycolide)/hydroxyapatite scaffolds loaded with bone mesenchymal stem cells and DNA complexes. Chin Sci Bull, 2012, 57: 435-444

11 Werner N, Kosiol S, Schiegl T, et al. Circulating endothelial progenitor cells and cardiovascular outcomes. N Engl J Med, 2005, 353: 999-1007

12 Levenberg S, Zoldan J, Basevitch Y, et al. Endothelial potential of human embryonic stem cells. Blood, 2007, 110: 806-814

13 Li Z, Han Z, Wu J C. Transplantation of human embryonic stem cellderived endothelial cells for vascular diseases. J Cell Biochem, 2009, 106: 194-199 
14 Li Z, Wilson K D, Smith B, et al. Functional and transcriptional characterization of human embryonic stem cell-derived endothelial cells for treatment of myocardial infarction. PLoS One, 2009, 4: e844

15 Huang N F, Niiyama H, Peter C, et al. Embryonic stem cell-derived endothelial cells engraft into the ischemic hindlimb and restore perfusion. Arterioscler Thromb Vasc Biol, 2010, 30: 984-991

16 Oyamada N, Itoh H, Sone M, et al. Transplantation of vascular cells derived from human embryonic stem cells contributes to vascular regeneration after stroke in mice. J Transl Med, 2008, 6: 54

17 Li Z, Wu J C, Sheikh A Y, et al. Differentiation, survival, and function of embryonic stem cell derived endothelial cells for ischemic heart disease. Circulation, 2007, 116: I46-54

18 Cho S W, Moon S H, Lee S H, et al. Improvement of postnatal neovascularization by human embryonic stem cell derived endotheliallike cell transplantation in a mouse model of hindlimb ischemia. Circulation, 2007, 116: 2409-2419

19 Uchimura H, Marumo T, Takase O, et al. Intrarenal injection of bone marrow-derived angiogenic cells reduces endothelial injury and mesangial cell activation in experimental glomerulonephritis. J Am Soc Nephrol, 2005, 16: 997-1004

20 Gnecchi M, Zhang Z, Ni A, et al. Paracrine mechanisms in adult stem cell signaling and therapy. Circ Res, 2008, 103: 1204-1219

21 Sangidorj O, Yang S H, Jang H R, et al. Bone marrow-derived endothelial progenitor cells confer renal protection in a murine chronic renal failure model. Am J Physiol Renal Physiol, 2010, 299: F325-335

22 Li Z, Suzuki Y, Huang M, et al. Comparison of reporter gene and iron particle labeling for tracking fate of human embryonic stem cells and differentiated endothelial cells in living subjects. Stem Cells, 2008, 26: 864-873

23 Li Z, Lee A, Huang M, et al. Imaging survival and function of transplanted cardiac resident stem cells. J Am Coll Cardiol, 2009, 53: 1229-1240

24 Yu J, Huang N F, Wilson K D, et al. nAChRs mediate human em- bryonic stem cell-derived endothelial cells: Proliferation, apoptosis, and angiogenesis. PLoS One, 2009, 4: e7040

25 Kwon O, Miller S, Li N, et al. Bone marrow-derived endothelial progenitor cells and endothelial cells may contribute to endothelial repair in the kidney immediately after ischemia-reperfusion. J Histochem Cytochem, 2010, 58: 687-694

26 Brodsky S V, Yamamoto T, Tada T, et al. Endothelial dysfunction in ischemic acute renal failure: Rescue by transplanted endothelial cells. Am J Physiol Renal Physiol, 2002, 282: F1140-1149

27 Kofidis T, Lebl D R, Martinez E C, et al. Novel injectable bioartificial tissue facilitates targeted, less invasive, large-scale tissue restoration on the beating heart after myocardial injury. Circulation, 2005, 112: I173-177

28 Frid M G, Kale V A, Stenmark K R. Mature vascular endothelium can give rise to smooth muscle cells via endothelial-mesenchymal transdifferentiation: In vitro analysis. Circ Res, 2002, 90: 1189-1196

29 Liebner S, Cattelino A, Gallini R, et al. Beta-catenin is required for endothelial-mesenchymal transformation during heart cushion development in the mouse. J Cell Biol, 2004, 166: 359-367

30 Sallustio F, De Benedictis L, Castellano G, et al. TLR2 plays a role in the activation of human resident renal stem/progenitor cells. FASEB J, 2010, 24: 514-525

31 Parikh C R, Lu J C, Coca S G, et al. Tubular proteinuria in acute kidney injury: A critical evaluation of current status and future promise. Ann Clin Biochem, 2010, 47: 301-312

32 Yeagy B A, Cherqui S. Kidney repair and stem cells: A complex and controversial process. Pediatr Nephrol, 2011, 26: 1427-1434

33 Dekel B, Zangi L, Shezen E, et al. Isolation and characterization of nontubular sca-1+lin- multipotent stem/progenitor cells from adult mouse kidney. J Am Soc Nephrol, 2006, 17: 3300-3314

34 Burchfield J S, Dimmeler S. Role of paracrine factors in stem and progenitor cell mediated cardiac repair and tissue fibrosis. Fibrogenesis Tissue Repair, 2008, 1: 4

Open Access This article is distributed under the terms of the Creative Commons Attribution License which permits any use, distribution, and reproduction in any medium, provided the original author(s) and source are credited. 